\title{
IMPLICATIONS OF BINARY PROPERTIES FOR THEORIES OF STAR FORMATION ${ }^{1}$
}

\author{
Richard B. Larson \\ Yale Astronomy Department \\ New Haven, CT 06520-8101, USA \\ larson@astro.yale.edu
}

\begin{abstract}
The overall frequency and other statistical properties of binary systems suggest that star formation is intrinsically a complex and chaotic process, and that most binaries and single stars actually originate from the decay of multiple systems. Interactions between stars forming in close proximity to each other may play an important role in the star formation process itself, for example via tidally induced accretion from disks. Some of the energetic activity of newly formed stars could be due to bursts of rapid accretion triggered by interactions with close companions.
\end{abstract}

\section{Introduction}

What can the study of binary stars tell us about how stars form? Stars and stellar systems can be viewed as fossils that preserve some record of the star formation process, and we wish to understand what they can tell us about this process. Single stars have only one parameter that is conserved from their time of formation, namely their mass, so the main thing we can hope to learn about star formation from single stars is the stellar initial mass function, which may constrain the origin of stellar masses. Binary systems have three additional conserved parameters, namely their angular momentum, eccentricity, and mass ratio, and therefore the statistical properties of binaries can in principle place stronger constraints on the nature of the star formation process. This is especially true concerning the small-scale dynamics of star formation and the mechanisms by which matter actually becomes incorporated into stars.

Most fundamentally, the frequency of binary and multiple systems tells us that most if not all stars are formed in such systems and not in isolation. The 'standard model' that has been developed to describe the formation in isolation of single stars like the Sun therefore cannot apply to most stars. Clearly the Sun is not typical in being a single star, but even the Sun could have formed in a multiple system because the plane of its planetary system is tilted with respect to the solar equatorial plane, plausibly because of a close encounter with another star soon after the Sun was formed (Herbig \& Terndrup 1986; Heller 1993). Therefore, not only is the formation of binary

1 Presented at IAU Symposium 200, "The Formation of Binary Stars", held in Potsdam, Germany, April 10-15, 2000; to be published by the Astronomical Society of the Pacific, edited by R. D. Mathieu and H. Zinnecker 
and multiple systems clearly nature's preferred way of making stars, it might even be nature's only way of making stars. This possibility would be appealing theoretically because if much of the angular momentum of a collapsing cloud goes into the orbital motion of a binary or multiple system, this would go a long way toward solving the classical 'angular momentum problem' of star formation.

A further basic fact about binary stars is that their orbital parameters vary over enormous ranges and show no clearly preferred values. This means that no 'standard model' of binary formation with any typical set of parameter values can adequately describe the formation of most binaries, or of most stars. A major conclusion to be elaborated below is that star formation must be, at least to some extent, intrinsically a chaotic process, involving complex dynamics and interactions in systems of forming stars, and that statistical approaches are needed to deal with the broad distribution of outcomes arising from such processes. Chaotic dynamics and protostellar interactions in forming binary and multiple systems may also have important implications for the nature of the accretion processes by which stars acquire most of their mass, and may account for some of the highly variable energetic activity observed in newly formed stars.

\section{Basic Statistical Properties of Binaries}

Although a clear consensus has not yet been reached on all of the statistical properties of binaries, the following basic properties seem reasonably clear:

(1) Frequency: The overall frequency of binaries, defined as the fraction of primaries that have at least one companion, is at least 50 percent (Heintz 1978; Abt 1983; Duquennoy \& Mayor 1991; Mayor et al. 1992). The binary fraction appears to increase with increasing primary mass, at least among the more massive stars: the $\mathrm{O}$ and $\mathrm{B}$ stars have a companion frequency of at least 70 percent (Abt, Gomez, \& Levy 1990; Mason et al. 1998; Preibisch et al. 1999; Preibisch 2001), while for G stars the binary frequency is around 50 percent (Duquennoy \& Mayor 1991; Fischer \& Marcy 1992) and the M stars may have an even lower binary frequency of around 30-40 percent (Fischer \& Marcy 1992; Tokovinin 1992; Mayor et al. 1992). Brown dwarfs are rare as companions to lower-main-sequence stars, although brown-dwarf binaries appear not to be rare (Basri 2001). An increase in binary frequency with mass would be expected if most stars form in multiple systems that disintegrate, since the more massive stars would then preferentially remain in binaries while the less massive ones would preferentially be ejected as single stars. The binary frequencies summarized above and their dependence on mass are in fact consistent with the results of simulations of the decay of small multiple systems (Sterzik \& Durisen 1998, 1999), and therefore they are consistent with the possibility that all stars are formed in such systems.

(2) Period Distribution: The periods of binaries are distributed continuously over an extremely large range (Heintz 1978; Abt 1983; Griffin 1992), and in a frequently quoted study Duquennoy \& Mayor (1991) found a broad and nearly flat distribution in the logarithm of the period which they fitted with a gaussian function centered on a median period of 180 years, corresponding to a median semi-major axis of about 35 AU. Pre-main-sequence stars show a very similar distribution of periods and separations (Mathieu 1994; Simon et al. 1995). The most remarkable feature of this distribution is its flatness, i.e. the fact that the number of systems per unit logarithmic interval is almost constant over many orders of magnitude in period or separation. Other authors have made the same point by noting that the distribution of semi-major axes $a$ follows the power-law form $f(a) \propto a^{-1}$, equivalent to a flat distribution in $\log a$, over many orders of magnitude in $a$ (Heacox 1998, 2000; Stepinski \& Black 2000a,b). This distribution is nearly scale-free and implies that there is no strongly preferred scale for the formation of binary systems. 
(3) Eccentricities: Binaries with periods longer than a year, which are not significantly affected by tidal circularization, have a broad distribution of orbital eccentricities $e$ that is nearly flat for $0<e<1$ (Aitken 1935; Duqennoy \& Mayor 1991; Mayor et al. 1992), with a median value of around 0.55. Clearly there is no tendency for binaries to form with nearly circular orbits, and high eccentricities are common; thus models of binary formation that postulate nearly circular orbits cannot adequately describe the formation of most systems.

(4) Mass Ratios: The distribution of mass ratios $q=M_{2} / M_{1}$ has been the most difficult function to pin down because it is subject to many biases and selection effects, and because it depends on period and probably also on primary mass. The strongest conclusion seems to be that the distribution of $q$ values is different for short-period and long-period binaries: according to Abt \& Levy (1978) (see also Abt 1983 and Abt \& Willmarth 1992), for systems with periods less than about 100 years (i.e., semi-major axes less than about $25 \mathrm{AU}$ ), the distribution of $q$ values is much flatter than would be predicted if the stars had been randomly selected from a standard IMF, while for longer-period systems the distribution of $q$ is more consistent with what would be predicted from the IMF. Mayor et al. (1992) and Mayor (2001) confirm that spectroscopic binaries have a distribution of $q$ values that is nearly flat in the range $0.2<q<1$, implying that the masses of the stars in these systems tend to be more nearly equal than would be predicted by random selection from the IMF.

As we have heard from Latham (2001), on the basis of impressive statistics, the statistical properties of binaries in the Galactic halo are in all respects indistinguishable from those of the binaries in the Galactic disk. Thus these properties are of great generality, and are not restricted to any particular place or time of formation.

\section{Implications for Theories of Star Formation}

From the above brief summary of the basic statistical properties of binaries, we can draw the following inferences for theories of star formation:

(1) At least two-thirds of all stars are in binary or multiple systems, and this can only be a lower limit to the fraction of stars formed in such systems. The statistical evidence summarized above is consistent with the possibility that all stars are formed in binary or multiple systems, and the minority of single stars result from the decay of multiple systems, as suggested by Heintz (1969) and Larson (1972). As an example, if stars typically form in triple systems that decay into a binary and a single star, this would yield similar numbers of binaries and single stars, as observed. Numerous simulations of the collapse and fragmentation of dense cloud cores, including many presented at this meeting, suggest that such multiple fragmentation processes are a very general result (Bodenheimer et al. 2000; Bodenheimer 2001; Bonnell 2001; Boss 2001; Klein 2001; Whitworth 2001). If so, a separate mechanism for forming single stars is not required.

(2) Given the above median orbital parameters, a typical star forms with a companion in an orbit having a period of about 180 years, a semi-major axis of about $35 \mathrm{AU}$, and an eccentricity of about 0.55 . The period and size of this 'median orbit' are similar to those of the planet Neptune, but this orbit is quite eccentric, unlike that of Neptune, and the separation of the two stars varies from about 16 to $54 \mathrm{AU}$. The formation of a planetary system similar to our own is clearly not possible such a situation, and any remaining circumstellar disk will be strongly disturbed by the tidal effect of the companion at every periastron passage. Circumstellar disks may be even more strongly perturbed for stars that form in multiple systems. 
(3) Since the orbital parameters of binaries vary widely, the detailed circumstances of star formation will also vary greatly from case to case, and most stars will not form in circumstances very similar to the above 'typical' case. Thus, there can be no 'standard model' for binary formation, or for the formation of stars generally, and a more statistical approach to the problem is needed. For example, instead of continually refining the accuracy of simulations of one or a few special cases, it may be more useful for future theoretical work to explore with less precision a larger parameter space and to try to predict the statistical distribution of outcomes.

(4) Since the stars in systems with separations smaller than a few tens of AU tend to have masses that are more nearly equal than would be predicted if they had been randomly selected from the IMF, the masses of stars that form within a few tens of AU of each other are correlated. This means that the mechanisms that determine stellar masses cannot be purely local to the star, and that effects acting on scales at least as large as a few tens of AU must play a role. The observed correlation could not be accounted for if, for example, stars "determine their own masses" by feedback effects that are purely local to the star and that act independently of the larger-scale environment. Stars must know something about the environment in which they form.

(5) The fact that the more massive stars tend to have more numerous and more massive companions suggests that interactions with companions play an increasingly important role in the formation of the more massive stars. It has been suggested that accretional processes associated with interactions, perhaps even including direct stellar collisions and coalescence, may play important roles in the formation of the most massive stars (Bonnell, Bate, \& Zinnecker 1998; Stahler, Palla, \& Ho 2000) and in the origin of the upper IMF (Larson 1999; Bonnell 2000), and these suggestions receive support from the observed higher frequency of companions among massive stars.

The following sections will consider further some of the above implications of the statistical properties of binaries for star formation, especially the effects of tidal interactions on disks in binary systems and the role of the chaotic dynamics of systems of forming stars in explaining the broad distribution of binary orbital parameters.

\section{Effect of Companions on Disk Evolution}

Numerical simulations of star formation often produce circumstellar disks, and remnant disks are also observed to be common around newly formed stars, even in binary systems (Mathieu 1994; Mathieu et al. 2000). A circumstellar disk in a binary system whose separation is not much larger than the size of the disk will be strongly tidally perturbed by the companion every time it passes periastron, and simulations show that these perturbations generate strong two-armed trailing spiral structure in the disk (Bate 2000, 2001; Nelson 2000; Nelson, Benz, \& Ruzmaikina 2000). If a tidally perturbed disk is continually replenished with new material, for example from an infalling envelope, the size and mass of the disk remain roughly constant and the amplitude of the tidally generated spiral pattern therefore also remains roughly constant in time, although the form of the pattern continually fluctuates. Such tidally produced spiral patterns are at least partly wave-like in nature, and they tend to propagate inward and dissipate in the inner part of the disk.

Tidally generated spiral waves may play an important role in driving accretion flows in disks (Spruit et al. 1987; Larson 1989; Savonije, Papaloizou, \& Lin 1994). A trailing spiral wave propagating into a disk has negative angular momentum, and thus it temporarily reduces the angular momentum of the disk; if the wave is somehow dissipated, the angular momentum of the disk is permanently reduced, and this can drive an inflow. For strong waves, a likely dissipation mechanism is the formation of shocks, and it was first suggested by Shu (1976) that spiral shocks might drive accretion flows in disks. Numerical simulations showing that tidally generated spiral 
shocks can indeed drive strong inflows in disks were presented by Sawada, Matsuda, \& Hachisu (1986) and Sawada et al. (1987), and self-similar solutions for shock-driven accretion were obtained by Spruit (1987). The disks studied by Sawada et al. $(1986,1987)$ were two-dimensional and had unrealistically high temperatures, but spiral shocks are also found in recent three-dimensional simulations, where these shocks are more tightly wound and resemble those inferred to exist in some cataclysmic variable systems (Haraguchi, Boffin, \& Matsuda 1999; Makita, Miyawaki, \& Matsuda 2000; Matsuda et al. 2000).

Accretion flows driven by tightly wound waves are a relatively weak effect, and therefore they are difficult to study numerically. Wave theory may then prove useful, and wave profiles for nonlinear acoustic waves in disks have been calculated by Larson (1990a) in order to determine the associated accretion rates. As with water waves, the wave profile is sinusoidal for small amplitudes but becomes increasingly sharply peaked at its crest as the amplitude increases, eventually 'breaking' to form a discontinuity or shock when the amplitude exceeds a critical value. Density profiles of the predicted form with inward-propagating shocks are seen in the high-resolution simulations of Różyczka \& Spruit (1993), which also show that the resulting wave pattern is often complex and time-dependent. The value of the Shakura-Sunyaev alpha parameter for a steady spiral wave pattern containing shocks can be estimated from the results of Larson (1990a) to be of the order of $(3-10) \times 10^{-4}$ for disks like those discussed here, implying an inflow timescale of the order of $(2-6) \times 10^{5}$ years at a radius of $1 \mathrm{AU}$. The inflow rate found numerically by Różyzcka \& Spruit (1993) corresponds to an average alpha of about $10^{-3}$, which is sufficient to drive significant inflows in circumstellar disks.

A full understanding of transport processes in disks does not yet exist, and several mechanisms could be involved. Wave effects will be important at least in the outer tidally disturbed parts of disks like those discussed here, where strong spiral waves are present. A trailing spiral wave pattern creates a gravitational torque that also acts to transport angular momentum outward (Lynden-Bell \& Kalnajs 1972; Larson 1984), but if the disk is of low mass and gravitationally stable, as most observed disks appear to be, the wave transport effect is more important than the gravitational torque for the same spiral pattern (Larson 1989). The ultimate fate of tidal waves propagating into a circumstellar disk is not yet clear, and it depends on how and where they are dissipated. If the waves are strongly damped at some radius, inflowing matter may tend to pile up there unless other transport mechanisms take over. If no other effect becomes more important, the continuing accumulation of matter at any radius will eventually cause gravitational instability to occur and gravitational torques to become important (Larson 1984; Stahler 2000), so inflow of matter seems likely to continue through one mechanism or another even if the tidal waves do not propagate all the way to the center.

Tidal effects can be particularly strong in very eccentric binaries, where they may cause episodes of rapid accretion at each periastron passage; this is suggested by the simulations of Bonnell \& Bastien (1992) in which violent spiral disturbances are generated at each periastron passage and lead to bursts of rapid accretion. Several transport mechanisms may play a role in these accretion events, since in addition to generating the wave effects discussed above, strong tidal disturbances may also result in enhanced gravitational and turbulent transport effects (Nelson et al. 2000). Even more violent disturbances that disrupt disks and/or trigger bursts of rapid accretion may be produced by close encounters in multiple systems, as is again illustrated by simulations of such encounters (Heller 1995; Boffin et al. 1998; Pfalzner, Henning, \& Kley 2000). If interactions with close companions can indeed trigger episodes of rapid accretion onto forming stars, this could help to explain the intriguing associations that have been reported at this meeting between the presence of close companions and the occurrence of protostellar jets (Reipurth 2001) and extreme T Tauri activity (Mathieu 2001). The FU Orionis phenomenon may also be explainable in a similar way (Bonnell \& Bastien 1992). A possible analogy may be noted between these phenomena of early 
stellar evolution and nuclear activity in galaxies, since the most extreme forms of activity in galaxies are also caused by violent tidal interactions and mergers that drive strong inflows in disks (Larson 1994).

\section{Understanding the Broad Period Distribution}

The fact that binaries are distributed in a logarithmically nearly uniform way over many orders of magnitude in period and separation is a challenge to theories of star formation, since standard models would tend to predict characteristic values of these quantities, while the observations show no evidence for any preferred scales and suggest instead a nearly scale-free formation process. The scale-free nature of the distribution of separations is also evident from the form of the distribution of semi-major axes, which in linear units is $f(a) \propto a^{-1}$ (Heacox 1998, 2000; Stepinski \& Black 2000a,b), and from the dependence on angular separation of the average surface density of companions on the sky, which follows $\Sigma(\theta) \propto \theta^{-2}$ (Larson 1995; Simon 1997). In the latter representation of the data, it is striking that the companion surface density falls off much more steeply with separation for binaries than it does for the larger-scale clustering of young stars in regions of star formation (Larson 1995; Bate, Clarke, \& McCaughrean 1998). This suggests that different processes act on different scales to determine the spatial distribution of young stars, and that processes acting on the scale of binaries produce a large excess of close pairs compared with what would be predicted from an extrapolation of the larger-scale clustering of young stars.

This distinction between binary systems and the larger-scale clustering of young stars is reminiscent of the situation for galaxies, which are also much more compact in structure than would be predicted from their clustering properties. There is even a quantitative similarity between the distribution of separations of binaries and the spatial distribution of stars in elliptical galaxies: as was first found by Hubble (1930; see also Holmberg 1975), the surface brightnesses of elliptical galaxies fall off approximately as the inverse square of distance from the center, and this resembles the inverse-square dependence of the surface density of binary companions on separation. Although the light profiles of elliptical galaxies tend to become shallower with increasing luminosity (Schombert 1987), a power law of slope -2 remains a representative approximation for galaxies of intermediate luminosity. The stars in binary systems and the stars in elliptical galaxies are thus both distributed roughly uniformly with respect to the logarithm of separation or distance from the center of mass. Similar surface density profiles have been found for some young star clusters (Moffat, Drissen, \& Shara 1994), so it may be a general phenomenon that systems of stars tend to be formed with their stars distributed roughly uniformly in the logarithm of separation.

The centrally condensed structures of elliptical galaxies are believed to result from dissipative formation processes, which could involve either gaseous dissipation or dynamical friction effects or both (Kormendy 1990; Larson 1990c). The fact that binary systems are relatively tightly bound compared to larger groupings of young stars suggests that dissipative effects have played a role in their formation too (Larson 1997; Heacox 1998, 2000). Such effects generally involve the loss of both energy and angular momentum, but since the energy of a collapsing cloud is easily radiated away while its angular momentum is less easily disposed of, the angular momentum is probably the most important dynamical parameter determining the outcome of the collapse and the separation of any resulting binary. The median specific angular momentum of binary systems is about an order of magnitude smaller than that of dense cloud cores, so the formation of a typical binary from a typical cloud core must involve a reduction in specific angular momentum by about an order of magnitude (Bodenheimer 1995). In addition, the amount of angular momentum lost or redistributed during the formation process must also vary widely from case to case to account for the observed broad distribution of binary separations. 
If the period distribution found by Duquennoy \& Mayor (1991) is approximated by the gaussian function of the logarithm of the period suggested by these authors, the corresponding distribution of the specific angular momentum $j$ of binaries is a gaussian function of $\log j$ with a mean of about -3.6 and a width at half maximum of about 1.8 if $j$ is measured in $\mathrm{km} \mathrm{s}^{-1} \mathrm{pc}$ and logarithms to the base 10 are used. This is a much broader distribution than would be produced by any simple gaussian random process, since such a process would tend to generate a distribution that is gaussian in each of the three components of $j$ rather than one that is gaussian in $\log j$. For example, if each of the three components of $j$ has a gaussian distribution with a mean of zero and a standard deviation $\sigma$, the distribution of $\log j$ is proportional to $j^{3} \exp \left(-j^{2} / 2 \sigma^{2}\right)$ and has a width at half maximum of only 0.43 . The median initial value of $\log j$ suggested by the velocity gradients measured for cloud cores by Goodman et al. (1993) is about -2.7 , so if cores like these are to form binary systems with the observed distribution of properties, the median $\log j$ must be reduced from -2.7 to -3.6 . At the same time, any plausible initial distribution of $\log j$ must be broadened considerably; in the above example, it must be broadened by more than a factor of 4 from a width of 0.43 to a width of 1.8 at half maximum.

Since the mechanisms that form binaries must accordingly reduce $\log j$ by an amount that varies widely from case to case, we can regard the amount by which $\log j$ is reduced as a random variable which has a large dispersion. For example, if we denote by $X$ the amount by which the natural $\log$ arithm $\ln j$ is reduced from its initial value $\ln j_{0}$, we can write $j=j_{0} e^{-X}$ where $X$ is a random variable whose mean and standard deviation must be about 2.1 and 1.7 respectively to account for the observations. Such an exponential dependence of a physical quantity on a negative exponent is suggestive of a damping or decay process; a familiar astronomical example is interstellar extinction, whereby a moderate dispersion in optical depth $\tau$ produces a large dispersion in the apparent brightnesses of stars, which vary as $e^{-\tau}$. To illustrate how such an effect might operate on a dynamical variable like $j$, suppose that the angular momentum of a forming binary system is reduced by a decelerating torque or drag effect, which might be of gravitational, magnetic, or viscous origin, and suppose that its angular momentum has an associated decay rate $A$ such that $d j / d t=-A j$; then after any time $t$ we have $j=j_{0} e^{-A t}$, so that if either the magnitude $A$ of the drag effect or the time $t$ over which it operates varies significantly from case to case, a large logarithmic dispersion in specific angular momentum $j$ can be produced (Larson 1997; Heacox 1998).

Angular momentum is conserved only in an axisymmetric system, and gravitational torques will redistribute angular momentum whenever there are departures from axial symmetry; for example, the gravitational drag that acts on orbiting clumps in a fragmenting cloud tends to reduce their orbital angular momentum and cause them to form more tightly bound systems (Larson 1978, 1984). Boss (1984) showed that this effect can in some cases reduce the angular momentum of a forming binary system by a large factor within an orbital period (see also Boss 1988, 1993 and Bodenheimer 1995). This gravitational drag effect is closely analogous to the 'dynamical friction' of stellar dynamics (Binney \& Tremaine 1987), and it may play an important role not only in the formation of binary systems but also in the formation of clusters like the Trapezium cluster, which has at its center the compact and massive Trapezium multiple system (Larson 1990b; Zinnecker, McCaughrean, \& Wilking 1993).

Gravitational forces also vary with space and time in a system with a clumpy mass distribution, and a random element is introduced if the dynamics of the system becomes chaotic. Chaotic dynamics is indeed expected if stars typically form in multiple systems, as is suggested by many numerical simulations of collapsing and fragmenting clouds, including those of Burkert, Bate, \& Bodenheimer (1997; see also Bodenheimer et al. 2000) and those presented at this meeting by Bodenheimer, Bonnell, Boss, Klein, and Whitworth. In the limit where most of the mass has condensed into stars, close encounters between the stars in a forming multiple system will produce 
large perturbations in their orbital motions, and these perturbations will on the average tend to make the closer subsystems more tightly bound, as happens with binaries in star clusters (Heggie 1975). A young binary system may in this situation lose most of its angular momentum through a few close encounters with other stars. The overall loss in angular momentum implied by the median initial and final values of $j$ given above is a factor of 8 , and this could be achieved through a few encounters if, for example, each encounter reduces the angular momentum of the system by a factor of 2 and the average number of such encounters is 3. Random fluctuations in this small number of events could then account for much of the dispersion in the final orbital properties of binaries. The standard deviation in $X$ arising just from the square root of the number of events is 1.2 , but if different encounters vary in their effects by a similar amount, the total standard deviation in $X$ becomes about 1.7, as required.

To test whether such effects can account for the observed distributions of properties of binaries, numerical simulations of the formation of small multiple systems are needed that predict the properties of the resulting binaries, but most calculations have not been carried to the point where most of the mass is in stars, and therefore they do not yet predict the properties of the resulting systems. Among the few simulations that have been carried this far are the early crude ones of Larson (1978); although their accuracy is low, these simulations do include the gravitational effects discussed above, and these effects play an important role in producing the many binary and multiple systems that are illustrated for example in Figures 6(a) and 6(b) of Larson (1978). The clustering of these objects can be examined by plotting the average surface density of companions as a function of separation, as was done for T Tauri stars by Larson (1995), and the results are very similar: there is a distinct binary regime on small scales where the companion surface density falls off much more steeply with separation than on larger scales, again varying approximately as the -2 power of separation (Larson 1997). Although the range of separations covered by these simulations is small compared to that represented by the observations, the fact that these results resemble the observations so closely suggests that the period distribution of binaries results from basic and universal features of gravitational dynamics that are present even in these crude simulations.

The formation of elliptical galaxies has been modeled in much more detail than the formation of binary systems, and the results of this work may also be relevant here if general gravitational mechanisms are involved (Larson 1997). Elliptical galaxies are believed to be formed by mergers of smaller systems, or by the collapse of clumpy protogalaxies containing substructures that are eventually erased. In either case, the main effect responsible for producing the final centrally condensed structure of the system is probably dynamical friction acting on the densest subunits and causing them to sink toward the center. The merger simulations of White (1978), Villumsen (1982), and Barnes (1992) and the simulations of clumpy collapse by van Albada (1982) and Katz (1991) all yield similar results that reproduce approximately the observed structures of elliptical galaxies. In these simulations, different mass elements experience widely differing amounts of dynamical friction and energy loss, and this results in a mass distribution in which the mass is spread out roughly uniformly in logarithmic intervals of radius, as observed.

Similar effects may occur statistically in the much smaller systems considered here, leading again to a distribution of separations that is roughly uniform in the logarithm of separation. In the limit where all of the mass has condensed into stars and the system has become a small n-body system, simulations of the decay of small multiple systems such as those made by Sterzik \& Durisen $(1998,1999)$ become relevant. The results of many such simulations show that n-body dynamics can indeed create a wide spread in the separations of the resulting binaries, and that this can account for a good part of the observed spread of separations. However, the closest binaries are still not reproduced, and this suggests that strongly dissipative gas dynamical effects are needed to form these systems. Thus a combination of gas dynamical and stellar dynamical effects is probably required to account for the full range of binary properties: gas dynamics provides the 
strong dissipation via shock formation that is needed to make close binary systems and individual stars, while the stellar dynamical effects that dominate when the system becomes very clumpy introduce a chaotic element that leads to a large dispersion in the final results.

\section{Summary}

The statistical properties of binary systems all point to a more complex and dynamic picture of star formation than that provided by the standard models for isolated star formation that have dominated theoretical work so far. From the frequency of binary and multiple systems it is clear that stars seldom if ever form in isolation, and the wide dispersion in binary properties suggests that even binaries typically do not form in isolation but as parts of larger systems whose dynamics is complex. The dependence of binary frequency on mass is consistent with the possibility that all binaries and single stars originate from the decay of multiple systems; part of the wide range in binary properties may then result from the chaotic dynamics of such systems. Many simulations of the collapse and fragmentation of dense cloud cores suggest that the typical outcome is indeed the formation of multiple systems with chaotic dynamics. This occurs partly because realistic initial conditions always have some degree of irregularity that tends to be amplified during the collapse, and partly because gravitational and hydrodynamic instabilities generate additional structure during the collapse which ultimately results in chaotic behavior. Future theoretical work on star formation will therefore have to deal with chaotic systems and be able to predict a statistical distribution of outcomes.

This complexity of the dynamics also has implications for the mechanisms by which gas becomes incorporated into forming stars. In standard models, most of the matter that goes into a star is assumed to be acquired by accretion from a disk. Disks may also be involved in the formation of stars in binary and multiple systems, but in this case tidal effects may play an important role in the accretion process. In eccentric binaries and in multiple systems, close encounters may produce particularly violent tidal effects that lead to bursts of enhanced accretion, and the stars in such systems might acquire much of their mass as a result of such discrete accretion events. Some young stars show variable activity that might reflect episodes of enhanced accretion triggered by interactions with companions; the FU Orionis phenomenon and protostellar jets might have such origins, and there is evidence that both protostellar jets and extreme T Tauri activity are associated with the presence of close companions. It will be of great interest to try to establish by further research whether some of the more dramatic forms of activity in young stars actually reflect an intrinsically violent and chaotic star formation process.

\section{References}

Abt, H. A. 1983, ARA\&A, 21, 343

Abt, H. A., \& Levy, S. G. 1978, ApJS, 36, 241

Abt, H. A., Gomez, A. E., \& Levy, S. G. 1990, ApJS, 74, 551

Abt, H. A.,, \& Willmarth, D. W. 1992, in Complementary Approaches to Double and Multiple Star Research, IAU Colloq. 135, eds. H. A. McAlister \& W. I. Hartkopf, ASP Conference Series Vol. 32 , p. 82

Aitken, R. G. 1935, The Binary Stars (McGraw-Hill, New York; reprinted by Dover, 1964)

Barnes, J. E. 1992, ApJ, 393, 484

Basri, G. 2001, in The Formation of Binary Stars, IAU Symp. 200, eds. R. D. Mathieu \& H. Zinnecker (ASP, San Francisco), in press (this volume) 
Bate, M. R. 2000, MNRAS, 314, 33

Bate, M. R. 2001, in The Formation of Binary Stars, IAU Symp. 200, eds. R. D. Mathieu \& H. Zinnecker (ASP, San Francisco), in press (this volume)

Bate, M. R., Clarke, C. J., \& McCaughrean, M. J. 1998, MNRAS, 297, 1163

Binney, J., \& Tremaine, S. 1987, Galactic Dynamics (Princeton University Press, Princeton)

Bodenheimer, P. 1995, ARA\&A, 33, 199

Bodenheimer, P. 2001, in The Formation of Binary Stars, IAU Symp. 200, eds. R. D. Mathieu \& H. Zinnecker (ASP, San Francisco), in press (this volume)

Bodenheimer, P., Burkert, A., Klein, R. I., \& Boss, A. P. 2000, in Protostars and Planets IV, eds. V. Mannings, A. P. Boss, \& S. S Russell (University of Arizona Press, Tucson), p. 675

Boffin, H. M. J., Watkins, S. J., Bhattal, A. S., Francis, N., \& Whitworth, A. P. 1998, MNRAS, 300,1189

Bonnell, I. A. 2000, in Star Formation from the Small to the Large Scale, 33rd ESLAB Symposium, eds. F. Favata, A. A. Kaas, \& A. Wilson (ESA, Noordwijk; ESA SP-44), in press

Bonnell, I. A. 2001, in The Formation of Binary Stars, IAU Symp. 200, eds. R. D. Mathieu \& H. Zinnecker (ASP, San Francisco), in press (this volume)

Bonnell, I., \& Bastien, P. 1992, ApJ, 401, L31

Bonnell, I. A., Bate, M. R., \& Zinnecker, H. 1998, MNRAS, 298, 93

Boss, A. P. 1984, MNRAS, 209, 543

Boss, A. P. 1988, Comments Astrophys., 12, 169

Boss, A. P. 1993, in The Realm of Interacting Binary Stars, eds. J. Sahade, G. E. McCluskey, \& Y. Kondo (Kluwer, Dordrecht), p. 355

Boss, A. P. 2001, in The Formation of Binary Stars, IAU Symp. 200, eds. R. D. Mathieu \& H. Zinnecker (ASP, San Francisco), in press (this volume)

Burkert, A., Bate, M. R., \& Bodenheimer, P. 1997, MNRAS, 289, 497

Duquennoy, A., \& Mayor, M. 1991, A\&A, 248, 485

Fischer, D. A., \& Marcy, G. W. 1992, ApJ, 396, 178

Goodman, A. A., Benson, P. J., Fuller, G. A., \& Myers, P. C. 1993, ApJ, 406, 528

Griffin, R. F. 1992, in Complementary Approaches to Double and Multiple Star Research, IAU Colloq. 135, eds. H. A. McAlister \& W. I. Hartkopf, ASP Conference Series Vol. 32, p. 98

Haraguchi, K., Boffin, H. M. J., \& Matsuda, T. 1999, in Star Formation 1999, ed. T. Nakamoto (Nobeyama Radio Observatory, Nobeyama), p. 241

Heacox, W. D. 1998, AJ, 115, 325

Heacox, W. D. 2000, in Birth and Evolution of Binary Stars, poster proceedings of IAU Symp. 200, eds. B. Reipurth \& H. Zinnecker (AIP, Potsdam), p. 208

Heggie, D. C. 1975 , MNRAS, 173, 729

Heintz, W. D. 1969, JRASC, 63, 275

Heintz, W. D. 1978, Double Stars (Reidel, Dordrecht)

Heller, C. H. 1993, ApJ, 408, 337

Heller, C. H. 1995, ApJ, 455, 252

Herbig, G. H., \& Terndrup, D. M. 1986, ApJ, 307, 609

Holmberg, E. 1975, in Galaxies and the Universe, eds. A. Sandage, M. Sandage, \& J. Kristian (University of Chicago Press, Chicago), p. 123

Hubble, E. P. 1930, ApJ, 71, 231

Katz, N. 1991, ApJ, 368, 325

Klein, R. I. 2001, in The Formation of Binary Stars, IAU Symp. 200, eds. R. D. Mathieu \& H. Zinnecker (ASP, San Francisco), in press (this volume)

Kormendy, J. 1990, in Dynamics and Interactions of Galaxies, ed. R. Wielen (Springer-Verlag, Berlin), p. 499 
Larson, R. B. 1972, MNRAS, 156, 437

Larson, R. B. 1978, MNRAS, 184, 69

Larson, R. B. 1984, MNRAS, 206, 197

Larson, R. B. 1989, in The Formation and Evolution of Planetary Systems, eds. H. A. Weaver \& L. Danly (Cambridge University Press, Cambridge), p. 31

Larson, R. B. 1990a, MNRAS, 243, 588

Larson, R. B. 1990b, in Physical Processes in Fragmentation and Star Formation, eds. R. CapuzzoDolcetta, C. Chiosi, \& A. Di Fazio (Kluwer, Dordrecht), p. 389

Larson, R. B. 1990c, PASP, 102, 709

Larson, R. B. 1994, in Mass-Transfer Induced Activity in Galaxies, ed. I. Shlosman (Cambridge University Press, Cambridge), p. 489

Larson, R. B. 1995, MNRAS, 272, 213

Larson, R. B. 1997, in Structure and Evolution of Stellar Systems, eds. T. A. Agekian, A. A. Mülläri, \& V. V. Orlov (St. Petersburg University Press, St. Petersburg), p. 48

Larson, R. B. 1999, in Star Formation 1999, ed. T. Nakamoto (Nobeyama Radio Observatory, Nobeyama), p. 336

Latham, D. W. 2001, in The Formation of Binary Stars, IAU Symp. 200, eds. R. D. Mathieu \& H. Zinnecker (ASP, San Francisco), in press (this volume)

Lynden-Bell, D., \& Kalnajs, A. J. 1972, MNRAS, 157, 1

Makita, M., Miyawaki, K., \& Matsuda, T. 2000, MNRAS, in press

Mason, B. D., Gies, D. R., Hartkopf, W. I., Bagnuolo, W. G., ten Brummelaar, T., \& McAlister, H. A. 1998, AJ, 115, 821

Mathieu, R. D. 1994, ARA\&A, 32, 465

Mathieu, R. D. 2001, in The Formation of Binary Stars, IAU Symp. 200, eds. R. D. Mathieu \& H. Zinnecker (ASP, San Francisco), in press (this volume)

Mathieu, R. D., Ghez, A. M., Jensen, E. L. N., \& Simon, M. 2000, in Protostars and Planets IV, eds. V. Mannings, A. P. Boss, \& S. S Russell (University of Arizona Press, Tucson), p. 703

Matsuda, T., Makita, M., Fujiwara, H., Nagae, T., Haraguchi, K., Hayashi, E., \& Boffin, H. M. J. 2000, AP\&SS, in press

Mayor, M. 2001, in The Formation of Binary Stars, IAU Symp. 200, eds. R. D. Mathieu \& H. Zinnecker (ASP, San Francisco), in press (this volume)

Mayor, M., Duquennoy, A., Halbwachs, J.-L., \& Mermilliod, J.-C. 1992, in IAU Colloq. 135, Complementary Approaches to Double and Multiple Star Research, eds. H. A. McAlister \& W. I. Hartkopf, ASP Conference Series Vol. 32, p. 73

Moffat, A. F. J., Drissen, L., \& Shara, M. M. 1994, ApJ, 436, 183

Nelson, A. 2000, in Birth and Evolution of Binary Stars, poster proceedings of IAU Symp. 200, eds. B. Reipurth \& H. Zinnecker (AIP, Potsdam), p. 205

Nelson, A. F., Benz, W., \& Ruzmaikina, T. V. 2000, ApJ, 529, 357

Pfalzner, S., Henning, Th., \& Kley, W. 2000, in Birth and Evolution of Binary Stars, poster proceedings of IAU Symp. 200, eds. B. Reipurth \& H. Zinnecker (AIP, Potsdam), p. 193

Preibisch, T., Balega, Y., Hofmann, K.-H., Weigelt, G., \& Zinnecker, H. 1999, NewA, 4, 531

Preibisch, T. 2001, in The Formation of Binary Stars, IAU Symp. 200, eds. R. D. Mathieu \& H. Zinnecker (ASP, San Francisco), in press (this volume)

Reipurth, B. 2001, in The Formation of Binary Stars, IAU Symp. 200, eds. R. D. Mathieu \& H. Zinnecker (ASP, San Francisco), in press (this volume)

Różyczka, M., \& Spruit, H. C. 1993, ApJ, 417, 677

Savonije, G. J., Papaloizou, J. C. B., \& Lin, D. N. C. 1994, MNRAS, 268, 13

Sawada, K., Matsuda, T., \& Hachisu, I. 1986, MNRAS, 219, 75

Sawada, K., Matsuda, T., Inoue, M., \& Hachisu, I. 1987, MNRAS, 224, 307 
Schombert, J. M. 1987, ApJS, 64, 643

Shu, F. H. 1976, in Structure and Evolution of Close Binary Systems, IAU Symp. 73, eds. P. Eggleton, S. Mitton, \& J. Whelan (Reidel, Dordrecht), p. 253

Simon, M. 1997, ApJ, 482, L81

Simon, M., Ghez, A. M., Leinert, Ch., Cassar, L., Chen, W. P., Howell, R. R., Jameson, R. F., Matthews, K., Neugebauer, G., \& Richichi, A. 1995, ApJ, 443, 625

Spruit, H. C. 1987, A\&A, 184, 173

Spruit, H. C., Matsuda, T., Inoue, M., \& Sawada, K. 1987, MNRAS, 229, 517

Stahler, S. W. 2000, in Star Formation from the Small to the Large Scale, 33rd ESLAB Symposium, eds. F. Favata, A. A. Kaas, \& A. Wilson (ESA, Noordwijk; ESA SP-44), in press

Stahler, S. W., Palla, F., \& Ho, P. T. P. 2000, in Protostars and Planets IV, eds. V. Mannings, A. P. Boss, \& S. S. Russell (University of Arizona Press, Tucson), p. 327

Stepinski, T. F., \& Black, D. C. 2000a, A\&A, 356, 903

Stepinski, T. F., \& Black, D. C. 2000b, in Birth and Evolution of Binary Stars, poster proceedings of IAU Symp. 200, eds. B. Reipurth \& H. Zinnecker (AIP, Potsdam), p. 167

Sterzik, M. F., \& Durisen, R. H. 1998, A\&A, 339, 95

Sterzik, M. F., \& Durisen, R. H. 1999, in Star Formation 1999, ed. T. Nakamoto (Nobeyama Radio Observatory, Nobeyama), p. 387

Tokovinin, A. A. 1992, A\&A, 256, 121

van Albada, T. S. 1982, MNRAS, 201, 939

Villumsen, J. V. 1982, MNRAS, 199, 493

White, S. D. M. 1978, MNRAS, 184, 185

Whitworth, A. 2001, in The Formation of Binary Stars, IAU Symp. 200, eds. R. D. Mathieu \& H. Zinnecker (ASP, San Francisco), in press (this volume)

Zinnecker, H., McCaughrean, M. J., \& Wilking, B. A. 1993, in Protostars and Planets III, eds. E. H. Levy \& J. I. Lunine (University of Arizona Press, Tucson), p. 429 